\title{
CLASS $D$ SUPERMARTINGALES
}

\author{
BY GUY JOHNSON ${ }^{1}$ AND L. L. HELMS ${ }^{2}$
}

Communicated by J. L. Doob, June 18, 1962

The decomposition of a continuous parameter supermartingale into the difference of a martingale and a process with increasing sample functions has been studied by Meyer [1]. Meyer has shown that a non-negative uniformly integrable right-continuous (i.e., right-continuous sample functions) supermartingale $\left\{y_{t}: 0 \leqq t \leqq+\infty\right\}$ with $\lim _{t \rightarrow \infty} y_{t}=0$ can be decomposed in this manner if and only if the supermartingale is of class $D$ on $[0, \infty]$. Such a supermartingale is of class $D$ on $[0, \infty]$ if the family of random variables $\left\{y_{T}: T \in \Im\right\}$, where $\Im$ is the class of stopping times for the process, is uniformly integrable. Although Meyer has given some sufficient conditions under which a supermartingale is of class $D$ on $[0, \infty]$, the existence of supermartingales which are uniformly integrable but not of class $D$ on $[0, \infty]$ has not been settled. An example of a uniformly integrable supermartingale which is not of class $D$ on $[0, \infty]$ is given below. A necessary and sufficient condition for a supermartingale to be of class $D$ on $[0, \infty]$ is proven under the additional hypothesis that almost all sample functions of the process are continuous. This condition does not involve stopping times.

Let $(\Omega, F, P)$ denote a probability measure space. Points of $\Omega$ will be denoted by $\omega$. If $\left\{y_{t}, F_{t}: 0 \leqq t \leqq \infty\right\}$ is a supermartingale, a stopping time is a non-negative random variable $T$, which may take on the value $+\infty$, such that $\{\omega: T(\omega)<t\} \in F_{t}$ for every $t$. The class of such stopping times will be denoted by $\Im$. It is well known that $S \wedge T$, the infimum of the two stopping times $S$ and $T$, is a stopping time.

THEOREM. Let $\left\{y_{t}, F_{t}: 0 \leqq t \leqq \infty\right\}$ be a non-negative right-continuous supermartingale. If the family $\left\{y_{T}: T \in \Im\right\}$ is uniformly integrable, then $\lim _{n \rightarrow \infty} n P\left[\sup _{0 \leq t \leq \infty} y_{t}>n\right]=0$. The converse holds if the supermartingale has sample functions which are continuous with probability 1.

Proof. Let $y^{*}=\sup _{0 \leq t \geqq \infty} y_{t}$ and let $T_{n}(\omega)=\inf \left\{t: y_{t}(\omega) \geqq n\right\}$ (the infimum of the empty set is defined to be $+\infty)$. Then $T_{n} \in \Im$ for each positive integer $n$. For any $n \geqq \xi$,

$$
\int_{y_{T_{n}} \geq \xi} y_{T_{n}} d P \geqq \int_{y_{T_{n}} \geq n} y_{T_{n}} d P \geqq n P\left[y_{T_{n}} \geqq n\right] \geqq n P\left[y^{*}>n\right] .
$$

${ }^{1}$ Rice University and the University of Illinois. The research of the first author was supported by the Air Force Office of Scientific Research.

2 The work of the second author was supported by the National Science Foundation, NSF Grant 19869. 
This inequality along with the uniform integrability of the sequence $\left\{y_{T_{n}}\right\}$ implies that $\lim _{n \rightarrow \infty} n P\left[y^{*}>n\right]=0$. To prove the converse, suppose the supermartingale has sample functions which are continuous with probability 1 and $\lim _{n \rightarrow \infty} n P\left[y^{*}>n\right]=0$. Let $S$ be any stopping time in $\Im$. Using the right-continuity of the supermartingale, it is easily seen that the $y_{t}$-process is a supermartingale relative to the enlarged system of Borel fields $F_{t}^{+}=\bigcap_{s>t} F_{s}$. All stopping times in $\Im$ are strict stopping times relative to this enlarged system of Borel fields; i.e., if $T \in \Im$, then $\{\omega: T(\omega) \leqq t\} \in F_{t}^{+}$for all $t$. Since $S \wedge T_{n} \leqq S$, the two random variables $y_{S} \wedge_{T_{n}}$ and $y_{S}$ constitute a supermartingale relative to the enlarged system of Borel fields ( $[2$, p. 373] and, especially, the note on p. 379). Using the supermartingale inequality

$$
\int_{\nu_{S \wedge T_{n}>n-1}} y_{S \wedge T_{n}} d P \geqq \int_{y_{S \wedge T_{n}>n-1}} y_{S} d P \quad \text { for every } n \geqq 1 .
$$

Using this inequality, the equation

$$
\begin{aligned}
\int_{\nu_{S} \wedge T_{n}>n-1} y_{S \wedge T_{n}} d P= & \int_{\nu_{S \wedge T_{n}>n-1 ; S<T_{n}} y_{S \wedge T_{n}} d P} \\
& +\int_{\nu_{S} \wedge T_{n}>n-1 ; S \Sigma T_{n}} y_{S \wedge T_{n}} d P,
\end{aligned}
$$

and the fact that $S(\omega)<T_{n}(\omega)$ implies $y_{S \wedge T_{n}(\omega)}(\omega)<n$,

$$
\int_{y_{S} \wedge T_{n}>n-1} y_{S} d P \leqq n P\left[y_{S} \wedge T_{n}>n-1\right]+\int_{y_{T_{n}>n-1}} y_{T_{n}} d P
$$

The set appearing in the integral on the left side of this inequality can be replaced by $\left\{\omega: y_{S(\omega)}(\omega)>n-1\right\}$. To see this, note that $y_{S(\omega)}(\omega)>n-1$ implies $y_{S \wedge T_{n}(\omega)}(\omega)>n-1$ if $S(\omega) \leqq T_{n}(\omega)$; on the other hand, if $T_{n}(\omega)<S(\omega) \leqq+\infty$, then the sample function corresponding to $\omega$ must at some time exceed $n$ and $y_{T_{n}(\omega)}(\omega) \geqq n>n-1$. Moreover, since $y_{S \wedge T_{n}(\omega)}(\omega)>n-1$ implies $y^{*}(\omega)>n-1$, the first term on the right side can be replaced by $n P\left[y^{*}>n-1\right]$. In addition, the integral on the right side can be written as a sum of three integrals depending on whether $T_{n}=0,0<T_{n}<+\infty$, or $T_{n}=+\infty$. Thus,

$$
\begin{aligned}
\int_{y_{S}>n-1} y_{S} d P \leqq & n P\left[y^{*}>n-1\right]+\int_{y_{0}>n-1} y_{0} d P \\
& +\int_{y_{T}>n-1 ; 0<T_{n}<+\infty} y_{T_{n}} d P+\int_{y_{\infty}>n-1} y_{\infty} d P
\end{aligned}
$$


for $n \geqq 1$ and any stopping time $S$. Since almost all sample functions are continuous, $0<T_{n}(\omega)<+\infty$ implies that $y_{T_{n}(\omega)}(\omega)=n$ with probability 1 . Thus,

$$
\int_{\nu_{S}>n-1} y_{S} d P \leqq 2 n P\left[y^{*}>n-1\right]+\int_{y_{0}>n-1} y_{0} d P+\int_{v_{\infty}>n-1} y_{\infty} d P
$$

for any $n \geqq 1$ and any stopping time $S$. Since the right side of this inequality approaches zero as $n$ approaches infinity and does not involve stopping times,

$$
\lim _{n \rightarrow \infty} \int_{y_{S}>n} y_{S} d P=0 \quad \text { uniformly for } S \in \Im .
$$

This concludes the proof that the family $\left\{y_{T}: T \in \mathscr{Y}\right\}$ is uniformly integrable.

It is interesting to note that the uniform integrability of a nonnegative continuous supermartingale $\left\{y_{t}, F_{t}: 0 \leqq t \leqq+\infty\right\}$ is a consequence of the condition of the theorem imposed on the supremum of the process. It is evident from the above proof that the condition of the theorem is also sufficient without the continuity assumption if the sequence $\left\{y_{T_{n}}\right\}$ is uniformly integrable.

The following example shows that, in general, it is not possible to decompose a uniformly integrable continuous parameter supermartingale into a martingale and a process with increasing sample functions $\left[2\right.$, p. 353]. Consider a Brownian motion process $\left\{x_{t}: 0 \leqq t<+\infty\right\}$ in the three-dimensional Euclidean space starting from the point $p_{0}=(1,0,0)$ with associated probability measure $P$. The sample functions of such a process approach the point at infinity with probability 1 and are continuous with probability 1 . Consider also the superharmonic function $u(p)=1 / r$ where $r=\|p\|$ is the distance from $p$ to $\theta$ $=(0,0,0)$. Then $u\left(x_{t}\right)$ is a supermartingale with sample functions which are continuous with probability 1 [3]. Moreover, $\lim _{t \rightarrow \infty} u\left(x_{t}\right)$ $=0$ with probability 1 . Let $y_{t}=u\left(x_{t}\right), 0 \leqq t<+\infty$, and let $y_{\infty}=0$. The process $\left\{y_{t}: 0 \leqq t \leqq+\infty\right\}$ is a supermartingale. This process is also uniformly integrable. To prove this it suffices to show that $E\left[y_{t}\right]$ is a continuous function on $[0, \infty][2, p .359]$. This condition is satisfied since $E\left[y_{0}\right]=E\left[u\left(x_{0}\right)\right]=u\left(p_{0}\right)=1, E\left[y_{\infty}\right]=0$, and with the aid of an integral from potential theory $[4$, p. 82$]$

$$
E\left[y_{t}\right]=\frac{4 \pi}{(2 \pi t)^{3 / 2}}\left[\int_{0}^{1} r^{2} e^{-r^{2} / 2 t} d r+t e^{-1 / 2 t}\right]
$$

for $0<t<+\infty$. 
It will now be shown that the family of random variables $\left\{y_{T}: T \in \mathfrak{Y}\right\}$ is not uniformly integrable. For each $\alpha \in(0,1)$, let $T_{\alpha}$ denote the first time that the $y_{t}$-process hits the interval $[1 / \alpha,+\infty)$ whenever the process hits the interval. $T_{\alpha}$ is defined to be $+\infty$ whenever the process does not hit the interval $[1 / \alpha,+\infty)$. Equivalently, $T_{\alpha}$ is either the first time the $x_{t}$-process hits the closed ball $\bar{B}(\theta, \alpha)=\{p:\|p\| \leqq \alpha\}$ or $+\infty$ depending upon whether or not the process hits $\bar{B}(\theta, \alpha)$. Consider the family of random variables $\left\{y_{T_{\alpha}}: 0<\alpha<1\right\}$. Suppose $\xi>0$ and $1 / \alpha>\xi$. Then

$$
\int_{\nu_{T_{\alpha}}>\xi} y_{T_{\alpha}} d P=(1 / \alpha) P\left[x_{t} \text {-process hits } \bar{B}(\theta, \alpha)\right] \text {. }
$$

The probability on the right can be evaluated using harmonic measure [5]. Let $v(p)$ be the function which is 1 on $\{p:\|p\|=\alpha\}$, zero at infinity, and harmonic for $\alpha<\|p\|$. Then $v\left(p_{0}\right)$ is just the probability that a Brownian path starting from $p_{0}$ will hit $\bar{B}(\theta, \alpha)$ before hitting the point at infinity. Since $v(p)=\alpha /\|p\|$ for $\|p\|>\alpha, P\left[x_{t}\right.$-process hits $\bar{B}(\theta, \alpha)]=v\left(p_{0}\right)=\alpha /\left\|p_{0}\right\|=\alpha$ and

$$
\int_{y_{T_{\alpha}}>\xi} y_{T_{\alpha}} d P=1 \text { whenever } 1 / \alpha>\xi
$$

This proves that the family $\left\{y_{T_{\alpha}}: 0<\alpha<1\right\}$ is not uniformly integrable. Since this family is contained in the family of random variables $\left\{y_{T}: T \in \Im\right\}$, the latter family is not uniformly integrable. The above computation shows that the condition of the theorem is not satisfied. In fact, the probability that a Brownian path will hit the ball $\bar{B}(\theta, \alpha)$ is just the probability that the supremum of the $y_{t}$-process will exceed $1 / \alpha$. The computation shows that $(1 / \alpha) P\left[y^{*} \geqq 1 / \alpha\right]=1$.

\section{REFERENCES}

1. P. Meyer, $A$ decomposition theorem for supermartingales, Illinois J. Math. 6 (1962), 193-205.

2. J. L. Doob, Stochastic processes, Wiley, New York, 1953.

3. - Semimartingales and subharmonic functions, Trans. Amer. Math. Soc., 77 (1954), 86-121.

4. O. D. Kellogg, Foundations of potential theory, Dover, New York, 1953.

5. S. Kakutani, On Brownian motions in n-space, Proc. Imp. Acad. Tokyo 20 (1944), 648-652.

UNIVERSITY OF ILLINOIS 